International Journal of Pure and Applied Mathematics

Volume 105 No. 2 2015, 223-234

ISSN: 1311-8080 (printed version); ISSN: 1314-3395 (on-line version)

url: http://www.ijpam.eu

doi: http://dx.doi.org/10.12732/ijpam.v105i2.9

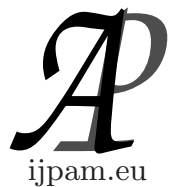

\title{
ON THE HYPERSURFACE OF A FINSLER SPACE WITH RANDERS CHANGE OF GENERALIZED $(\alpha, \beta)$ METRIC
}

\author{
Gauree Shanker ${ }^{1}$, Vijeta Singh ${ }^{2} \S$ \\ ${ }^{1,2}$ Department of Mathematics and Statistics \\ Banasthali University \\ Banasthali, Rajasthan, 304022, INDIA
}

\begin{abstract}
The study of special Finsler spaces has been introduced by M. Matsumoto [6]. The purpose of the present paper is to study hypersurfaces of a Finsler space with $(\alpha, \beta)$-metric $L^{*}=\alpha+\epsilon \beta+\kappa \frac{\beta^{2}}{\alpha}+\beta$. We have obtained the conditions for this hypersurface to be hyperplane of 1st, 2nd kind but not of $3 r d$ kind.
\end{abstract}

AMS Subject Classification: 53B40, 53C60

Key Words: finsler space, hypersurface, induced cartan connection, hyperplane of 1st, 2nd, 3rd kind

\section{Introduction}

Let $F^{n}=\left(M^{n}, L\right)$ be an n-dimensional Finsler space i.e. a pair consisting of an n-dimensional differential manifold $M^{n}$ equipped with a fundamental function $L(x, y)$. The concept of the $(\alpha, \beta)$-metric was introduced by M. Matsumoto [6] and has studied by many authors such as Shibata and others ([3], [5], [6], [8]), where $\alpha^{2}=a_{i j}(x) y^{i} y^{j}$ is a Riemannian metric and $\beta=b_{i}(x) y^{i}$ is a 1 -form on $M^{n}$. It has plenty of applications in various fields such as physics, mechanics, seismology, biology and ecology ([1], [2], [7], [10]).

Received: September 9, 2015

(C) 2015 Academic Publications, Ltd.

$\S$ Correspondence author url: www.acadpubl.eu 
A change of Finsler metric $L(x, y) \rightarrow L^{*}(x, y)=L(x, y)+b_{i}(x) y^{i}$ is called Randers change of metric. The notion of a Randers change ws proposed by Matsumoto, named by Hashiguchi and Ichijyo [4] and studied in detail by Shibata [9]. In the present paper we are introducing Randers change of general $(\alpha, \beta)$ metric, $L^{*}=\alpha+\epsilon \beta+\kappa \frac{\beta^{2}}{\alpha}+\beta$.

A hypersurface $M^{n-1}$ of the $M^{n}$ may be represented parametrically by the equation $x^{i}=x^{i}(u),=1, \ldots, n-1$, where $u$ are Gaussian coordinates on $M^{n-1}$. Since the function $L^{*}=(L(x(u), y(u, v))$ gives rise to a Finsler metric of $M^{n-1}$, we get an $(n-1)$-dimensional Finsler space $F^{*(n-1)}=\left(M^{n-1}, L^{*}(u, v)\right)$. The hypersurface of Finler Space with some given special metrics has been studied by authors.

In the present paper, we consider an n-dimensional Finsler space $F^{* n}=$ $\left(M^{n}, L^{*}\right)$ with $(\alpha, \beta)$-metric $L^{*}=\alpha+\epsilon \beta+\kappa \frac{\beta^{2}}{\alpha}+\beta$ and the hypersurface of $F^{n}$ with $b_{i}=\partial_{i} b$ being the gradient of a scalar function $b(x)$. We prove the conditions for this hypersurface to be a hyperplane of 1st kind, 2nd kind and we also prove that this hypersurface is not a hyperplane of 3rd kind.

\section{Preliminaries}

Let $M^{n}$ be a real smooth manifold of dimension $n$ and let $F^{* n}=\left(M^{n}, L^{*}\right)$ be a Finsler space on a differentiable manifold $M^{n}$ endowed with a fundamental function $L^{*}(x, y)$, where

$$
L^{*}=\alpha+\epsilon \beta+\kappa \frac{\beta^{2}}{\alpha}+\beta .
$$

The derivatives of the (2.1) with respect to $\alpha$ and $\beta$ is given by

$$
\begin{array}{ll}
L_{\alpha}^{*}=\frac{\alpha^{2}-\kappa \beta^{2}}{\alpha^{2}}, & L_{\beta}^{*}=\frac{(\epsilon+1) \alpha+2 \kappa \beta}{\alpha}, \\
L_{\alpha \alpha}^{*}=\frac{2 \kappa \beta^{2}}{\alpha^{3}}, & L_{\beta \beta}^{*}=\frac{2 \kappa}{\alpha}, \\
L_{\alpha \beta}^{*}=-\frac{2 \kappa \beta}{\alpha^{2}}, &
\end{array}
$$

where

$$
L_{\alpha}^{*}=\frac{\partial L^{*}}{\partial \alpha}, \quad L_{\beta}^{*}=\frac{\partial L^{*}}{\partial \beta}, \quad L_{\alpha \alpha}^{*}=\frac{\partial L_{\alpha}^{*}}{\partial \alpha}
$$




$$
L_{\beta \beta}^{*}=\frac{\partial L_{\beta}^{*}}{\partial \beta}, \quad L_{\alpha \beta}^{*}=\frac{\partial L_{\alpha}^{*}}{\partial \beta}
$$

In the special Finsler space $F^{* n}=\left(M^{n}, L^{*}\right)$ the normalized element of support $l_{i} \dot{\partial}_{i} L^{*}$ and the angular metric tensor $h_{i j}^{*}=L^{*} \dot{\partial}_{i} \dot{\partial}_{j} L^{*}$ :

$$
\begin{gathered}
l_{i}^{*}=\alpha^{-1} L_{\alpha}^{*} y_{i}+L_{\beta}^{*} b_{i}, \\
h_{i j}^{*}=p a_{i j}+q_{0} b_{i} b_{j}+q_{1}\left(b_{i} Y_{j}+b_{j} Y_{i}\right)+q_{2} Y_{i} Y_{j},
\end{gathered}
$$

where

$$
\begin{aligned}
Y_{i} & =a_{i j} y_{j} \\
p & =L^{*} L_{\alpha}^{*} \alpha^{-1}=\frac{\alpha^{4}+\alpha^{3} \beta(\epsilon+1)-\alpha \beta^{3} \kappa(\epsilon+1)-\kappa^{2} \beta^{4}}{\alpha^{4}}, \\
q_{0} & =L^{*} L_{\beta \beta}^{*}=\frac{2 \kappa\left\{\alpha^{2}+\alpha \beta(\epsilon+1)+\kappa \beta^{2}\right\}}{\alpha^{2}}, \\
q_{1} & =L^{*} L_{\alpha \beta}^{*} \alpha^{-1}=-\frac{2 \kappa \beta\left\{\alpha^{2}+\alpha \beta(\epsilon+1)+\kappa \beta^{2}\right\}}{\alpha^{4}}, \\
q_{2} & =L^{*} \alpha^{-2}\left(L_{\alpha \alpha}^{*}-L_{\alpha}^{*} \alpha^{-1}\right) \\
& =-\frac{\alpha^{4}+\alpha^{3} \beta(\epsilon+1)-2 \kappa \alpha^{2} \beta^{2}-3 \kappa(\epsilon+1) \alpha \beta^{3}-3 \kappa^{2} \beta^{4}}{\alpha^{6}} .
\end{aligned}
$$

The fundamental tensor $g_{i j}=(1 / 2) \dot{\partial}_{i} \dot{\partial}_{j} L^{* 2}$ is given by

$$
g_{i j}^{*}=p a_{i j}+p_{0} b_{i} b_{j}+p_{1}\left(b_{i} Y_{j}+b_{j} Y_{i}\right)+p_{2} Y_{i} Y_{j}
$$

where

$$
\begin{aligned}
& p_{0}=q_{0}+L_{\beta}^{* 2}=\frac{\alpha^{2}\left\{2 \kappa+(\epsilon+1)^{2}\right\}+\alpha\{6 \kappa(\epsilon+1) \beta\}+6 \kappa^{2} \beta^{2}}{\alpha^{2}}, \\
& p_{1}=q_{1}+L^{*-1} p L_{\beta}^{*}=\frac{(\epsilon+1) \alpha^{3}-3 \kappa(\epsilon+1) \alpha \beta^{2}-4 \kappa^{2} \beta^{3}}{\alpha^{4}}, \\
& p_{2}=q_{2}+p^{2} L^{*-2}=-\frac{(\epsilon+1) \alpha^{3}-3 \kappa(\epsilon+1) \alpha \beta^{3}-4 \kappa^{2} \beta^{4}}{\alpha^{6}} .
\end{aligned}
$$

Now, the reciprocal tensor $g^{* i j}$ of $g_{i j}^{*}$ is given by

$$
g^{* i j}=p^{-1} a^{i j}-s_{0} b^{i} b^{j}-s_{1}\left(b^{i} y^{j}+b^{j} y^{i}\right)-s_{2} y^{i} y^{j},
$$

where

$$
b^{i}=a^{i j} b_{j}
$$




$$
\begin{aligned}
s_{0} & =\frac{\left\{p p_{0}+\left(p_{0} p_{2}-p_{1}^{2}\right) \alpha^{2}\right\}}{\zeta p}, \\
s_{1} & =\frac{\left\{p p_{1}-\left(p_{0} p_{2}-p_{1}^{2}\right) \beta\right\}}{\zeta p}, \\
s_{2} & =\frac{\left\{p p_{2}+\left(p_{0} p_{2}-p_{1}^{2}\right) b^{2}\right\}}{\zeta p}, \quad b^{2}=a_{i j} b^{i} b^{j}, \\
\zeta=p\left(p+p_{0} b^{2}+p_{1} \beta\right) & +\left(p_{0} p_{2}-p_{1}^{2}\right)\left(\alpha^{2} b^{2}-\beta^{2}\right) .
\end{aligned}
$$

For general Finsler space $F^{n}$ the hv-torsion tensor is given by $C_{i j k}=p_{1}\left(h_{i j} m_{k}+\right.$ $\left.h_{j k} m_{i}+h_{k i} m_{j}\right)+\gamma_{1} m_{i} m_{j} m_{k}$, where

$$
\gamma_{1}=p \frac{\partial p_{0}}{\partial \beta}-3 p_{1} q_{0}, \quad m_{i}=b_{i}-\alpha^{-2} \beta Y_{i} .
$$

Here $m_{i}$ is a non-vanishing covariant vector orthogonal to the element of support $y^{i}$.

Let $\left\{\begin{array}{l}i \\ j k\end{array}\right\}$ be the component of Christoffel symbols of the associated Riemannian space $R^{n}$ and let $\nabla_{k}$ be covariant differentiation with respect to $x^{k}$ relative to this Christoffel symbols. We will use the following tensors:

$$
2 E_{i j}=b_{i j}+b_{j i}, \quad 2 F_{i j}=b_{i j}-b_{j i},
$$

where $b_{i j}=\nabla_{j} b_{i}$.

Let $C \Gamma=\left(F_{j k}^{i}, G_{j}^{i}, C_{j k}^{i}\right)$, be the Cartan connection of $F^{n}$. The difference tensor $D_{j k}^{i}=F_{j k}^{i}-\left\{\begin{array}{l}i \\ j k\end{array}\right\}$ of the special Finsler space $F^{n}$ is given by

$$
\begin{aligned}
D_{j k}^{i}= & B^{i} E_{j k}+F_{k}^{i} B_{j}+F_{j}^{i} B_{k}+B_{j}^{i} b_{0 k}+B_{k}^{i} b_{0 j}-b_{0 m} g^{i m} B_{j k} \\
& -C_{j m}^{i} A_{k}^{m}-C_{k m}^{i} A_{j}^{m}+C_{j k m} A_{s}^{m} g^{i s} \\
& +\lambda^{s}\left(C_{j m}^{i} C_{s k}^{m}+C_{k m}^{i} C_{s j}^{m}-C_{j k}^{m} C_{m s}^{i}\right),
\end{aligned}
$$

where:

$$
\begin{array}{ll}
B_{k}=p_{0} b_{k}+p_{1} y_{k}, & B^{i}=g^{i j} B_{j}, \\
F^{k}=g^{k j} F_{j i}, & B_{i j}=\frac{p_{1}\left(a_{i j}-\alpha^{-2} Y_{i} Y_{j}\right)+\left(\partial p_{0} / \partial \beta\right) m_{i} m_{j}}{2}, \\
\begin{array}{c}
A_{k}^{m}=B_{k}^{m} E_{00}+B^{m} E_{k 0} \\
\quad+B_{k} F_{0}^{m}+B_{0} F_{k}^{m},
\end{array} & B_{i}^{k}=g^{k j} B_{j i}, \\
\lambda^{m}=B^{m} E_{00}+2 B_{0} F_{0}^{m}, & B_{0}=B_{i} y^{i} .
\end{array}
$$

where 0 denote contraction with $y^{i}$ except for the quantities $p_{0}, q_{0}$ and $s_{0}$. 


\section{Induced Cartan Connection}

Let $F^{n-1}$ be a hypersurface of $F^{n}$ given by the equations $x^{i}=x^{i}(u)$. The element of support $y^{i}$ of $F^{n}$ is to be taken tangential to $F^{n-1}$, that is

$$
y^{i}=B_{\alpha}^{i}(u) v^{\alpha}
$$

The metric tensor $g_{\alpha \beta}$ and the v-torsion tensor $C_{\alpha \beta \gamma}$ are given by

$$
g_{\alpha \beta}=g_{i j} B_{\alpha}^{i} B_{\beta}^{j}, \quad C_{\alpha \beta \gamma}=C_{i j k} B_{\alpha}^{i} B_{\beta}^{j} B_{\gamma}^{k}
$$

At each point $u^{\alpha}$ of $F^{n-1}$, a unit normal vector $N^{i}(u, v)$ is defined by

$$
g_{i j} B_{\alpha}^{i} N^{j}=0, \quad \quad g_{i j} N^{i} N^{j}=1
$$

For the angular metric tensor $h_{i j}$, we have

$$
h_{\alpha \beta}=h_{i j} B_{\alpha}^{i} B_{\beta}^{j}, \quad h_{i j} B_{\alpha}^{i} N^{j}=0, \quad h_{i j} N^{i} N^{j}=1
$$

The inverse projection factors $B_{\alpha}^{i}(u, v)$ of $B_{\alpha}^{i}$ are defined as

$$
B_{i}^{\alpha}=g^{\alpha \beta} g_{i j} B_{\beta}^{j},
$$

where $g^{\alpha \beta}$ is the inverse of the metric tensor $g_{\alpha \beta}$ of $F^{n-1}$.

From (3.3) and (3.5), it follows that

$$
B_{\alpha}^{i} B_{i}^{\beta}=\delta_{\alpha}^{\beta}, \quad B_{\alpha}^{i} N_{i}=0, \quad N^{i} B_{i}^{\alpha}=0, \quad N^{i} N_{i}=1
$$

and further

$$
B_{\alpha}^{i} B_{j}^{\alpha}+N^{i} N_{j}=\delta_{j}^{i}
$$

For induced Cartan connection $I C \Gamma=\left(F_{\beta \gamma}^{\alpha}, G_{\beta}^{\alpha}, C_{\beta \gamma}^{\alpha}\right)$ on $F^{n-1}$, the second fundamental h-tensor $H_{\alpha \beta}$ and the normal curvature vector $H_{\alpha}$ are given by

$$
H_{\alpha \beta}=N_{i}\left(B_{\alpha \beta}^{i}+F_{j k}^{i} B_{\alpha}^{j} B_{\beta}^{k}\right)+M_{\alpha} H_{\beta}, \quad H_{\alpha}=N_{i}\left(B_{0 \alpha}^{i}+G_{j}^{i} B_{\alpha}^{j}\right),
$$

where $M_{\alpha}=C_{i j k} B_{\alpha}^{i} N^{j} N^{k}, B_{\alpha \beta}^{i}=\partial^{2} x^{i} / \partial u^{\alpha} \partial u^{\beta}$, and $B_{0 \alpha}^{i}=B_{\beta \alpha}^{i} v^{\beta}$. It is clear that $H_{\alpha \beta}$ is not symmetric and

$$
H_{\alpha \beta}-H_{\beta \alpha}=M_{\alpha} H_{\beta}-M_{\beta} H_{\alpha}
$$


Equation (3.8) yields

$$
H_{0 \alpha}=H_{\beta \alpha} v^{\beta}=H_{\alpha}, \quad H_{\alpha 0}=H_{\alpha \beta} v^{\beta}=H_{\alpha}+M_{\alpha} H_{0}
$$

The second fundamental v-tensor $M_{\alpha \beta}$ is defined as:

$$
M_{\alpha \beta}=C_{i j k} B_{\alpha}^{i} B_{\beta}^{j} N^{k}
$$

The relative h- and v- covariant derivatives of $B_{\alpha}^{i}$ and $N^{i}$ are given by

$$
B_{\alpha \mid \beta}^{i}=H_{\alpha \beta} N^{i},\left.\quad B_{\alpha}^{i}\right|_{\beta}=M_{\alpha \beta} N^{i}, \quad N_{\mid \beta}^{i}=-H_{\alpha \beta} B_{j}^{\alpha} g^{i j},\left.\quad N^{i}\right|_{\beta=-M_{\alpha \beta}} B_{j}^{\alpha} g^{i j} .
$$

Let $X_{i}(x, y)$ be a vector field of $F^{n}$, the relative h- and v-covriant derivatives of $X_{i}$ are given by

$$
X_{i \mid \beta}=X_{i \mid j} B_{\beta}^{j}+X_{i \mid j} N^{j} H_{\beta}, \quad X_{i \mid \beta}=\left.X_{i}\right|_{j} B_{\beta}^{j} .
$$

Matsumoto [3] defined different kind of hyperplanes and obtained their characterstics conditions, which are given in the following lemmas.

Lemma 3.1. For Finsler space $F^{n}$ a hypersurface $F^{n-1}$ is a hyperplane of the first kind if and only if $H_{\alpha}=0$ or equivalently $H_{0}=0$.

Lemma 3.2. For Finsler space $F^{n}$ a hypersurface $F^{n-1}$ is a hyperplane of the second kind if and only if $H_{\alpha \beta}=0$.

Lemma 3.3. For Finsler space $F^{n}$ a hypersurface $F^{n-1}$ is a hyperplane of the third kind if and only if $H_{\alpha \beta}=0=M_{\alpha \beta}$.

\section{Hypersurface $F^{n-1}$ of the Finsler Space with Randers Change of

$$
\text { General Metric } L=\alpha+\epsilon \beta+\kappa \frac{\beta^{2}}{\alpha}
$$

Let us consider a special Finsler metric $L^{*}=\alpha+\epsilon \beta+\kappa \frac{\beta^{2}}{\alpha}+\beta$ with a gradient $b_{i}(x)=\partial_{i} b$.

From parametric equation $x^{i}=x^{i}\left(u^{\alpha}\right)$ of $F^{n-1}(c)$, we get $\partial_{\alpha} b(x(u))=0=$ $b_{i} B_{\alpha}^{i}$ so that $b_{i}(x)$ are regarded as covariant components of a normal vector field of $F^{n-1}(c)$. Therefore, along the $F^{* n-1}(c)$ we have

$$
b_{i} B_{\alpha}^{* i}=0, \quad b_{i} y^{i}=0 .
$$


Therefore induced metric $L^{*}(u, v)$ of $F^{* n-1}(c)$ is given by

$$
L^{*}(u, v)=\sqrt{a_{\alpha \beta} v^{\alpha} v^{\beta}}, \quad a_{\alpha \beta}=a_{i j} B_{\alpha}^{* i} B_{\beta}^{* j}
$$

which is a Riemannian metric.

At a point of $F^{*(n-1)}(c)$, from $(2.5),(2.7)$ and (2.9), we have

$$
\begin{array}{llll}
p=1, & q_{0}=2 \kappa, & q_{1}=0, & q_{2}=-\frac{1}{\alpha}^{2} \\
p_{0}=2 \kappa+(\epsilon+1)^{2}, & p_{1}=\frac{\epsilon+1}{\alpha}, & p_{2}=0, & \zeta=1+2 \kappa b^{2}, \\
s_{0}=\frac{2 \kappa}{1+2 \kappa b^{2}}, & s_{1}=\frac{\epsilon+1}{\alpha\left(1+2 \kappa b^{2}\right)}, & s_{2}=-\frac{(\epsilon+1)^{2} b^{2}}{\alpha^{2}\left(1+2 \kappa b^{2}\right)}
\end{array}
$$

Therefore (2.8) gives

$$
g^{* i j}=a^{i j}-\frac{2 \kappa}{1+2 \kappa b^{2}} b^{i} b^{j}-\frac{(\epsilon+1)}{\alpha\left(1+2 \kappa b^{2}\right)}\left(b^{i} y^{j}+b^{j} y^{i}\right)+\frac{(\epsilon+1)^{2} b^{2}}{\alpha^{2}\left(1+2 \kappa b^{2}\right)},
$$

Using (4.1), we get

$$
g^{* i j} b_{i} b_{j}=\frac{b^{2}}{1+2 \kappa b^{2}}
$$

which gives

$$
b_{i}(x(u))=\sqrt{\frac{b^{2}}{1+2 \kappa b^{2}}} N_{i}^{*}
$$

where $b$ is the length of the vector $b^{i}$. Using (4.1) and (4.6) we get

$$
b^{i}=a^{i j} b_{j}=\sqrt{b^{2}\left(1+2 \kappa b^{2}\right)} N^{* i}+(\epsilon+1) b^{2} \alpha^{-1} y^{i} .
$$

Theorem 4.1. In the special Finsler hypersurface $F^{*(n-1)}(c)$, the induced metric is a Riemannian metric given by (4.2) and the scalar function $b(x)$ is given by (4.5)and (4.6).

The angular metric tensor and metric tensor of $F^{* n}$ are given by

$$
\begin{array}{r}
h_{i j}^{*}=a_{i j}+2 \kappa b_{i} b_{j}-\frac{y_{i} y_{j}}{\alpha^{2}}, \\
g_{i j}^{*}=a_{i j}+\left[2 \kappa+(\epsilon+1)^{2}\right] b_{i} b_{j}+\frac{(\epsilon+1)}{\alpha}\left(b_{i} y_{j}+b_{j} y_{i}\right) .
\end{array}
$$


If $h_{\alpha \beta}^{(a)}$ denote angular metric tensor of the Riemmanian metric $a_{i j}(x)$ then, using (4.1),(4.8) and (3.4) for induced metric $L^{*}$, we get

$$
h_{\alpha \beta}^{*}=h_{\alpha \beta}^{*(a)} .
$$

From (2.7), we get

$$
\frac{\partial p_{0}}{\partial \beta}=\frac{6 \kappa(\epsilon+1)}{\alpha}+\frac{12 \kappa^{2} \beta^{2}}{\alpha^{2}}
$$

Thus, along $F^{* n-1)}(c), \frac{\partial p_{0}}{\partial \beta}=\frac{6 \kappa(\epsilon+1)}{\alpha}$, and therefore (2.10) gives $\gamma_{1}=$ $0, m_{i}=b_{i}$.

Then the hv-tensor becomes

$$
C_{i j k}^{*}=\frac{(\epsilon+1)}{2 \alpha}\left(h_{i j} b_{k}+h_{j} k b_{i}+h_{k i} b_{j}\right),
$$

and therefore using (3.4), (3.11) and (4.1), we get

$$
M_{\alpha \beta}^{*}=\left(\frac{\epsilon+1}{2 \alpha}\right) \sqrt{\frac{b^{2}}{1+2 \kappa b^{2}}} h_{\alpha \beta}^{*}, \quad M_{\alpha}^{*}=0
$$

and hence from (3.9) it follows that $H_{\alpha \beta}^{*}$ is symmetric. Thus we have the following.

Theorem 4.2. The second fundamental $v$ tensor of special Finsler hypersurface $F^{* n-1)}(c)$ vanishes and the second fundamental $h$ - tensor $H_{\alpha \beta}^{*}$ is symmetric.

Next taking h-covariant derivative of (4.1) with respect to the induced connection, we get

$$
b_{i \mid \beta} B_{\alpha}^{* i}+b_{i} B_{\alpha \mid \beta}^{* i}=0
$$

Applying (3.13) for the vector $b_{i}$, we get

$$
b_{i \mid \beta} B_{\alpha}^{* i}=b_{i \mid j} B_{\beta}^{* j}+b_{i \mid j} N^{* j} H_{\beta}^{*} .
$$

Using this and $B_{\alpha \mid \beta}^{* i}=H_{\alpha \beta}^{*} N^{* i},(4.14)$ becomes

$$
b_{i \mid j} B_{\alpha}^{* i} B_{\beta}^{* j}+\left.b_{i}\right|_{j} B_{\alpha}^{* i} N^{* j} H_{\beta}^{*}+b_{i} H_{\alpha \beta}^{*} N^{* i}=0
$$


Since $\left.b_{i}\right|_{j}=-b_{h} C_{i j}^{h}$ for Finsler space $F^{n}$, using (4.6) and (4.13), we get

$$
\left.b_{i}\right|_{j} B_{\alpha}^{* i} N^{* j}=-\sqrt{\frac{b^{2}}{1+2 \kappa b^{2}}} M_{\alpha}^{*}=0
$$

Thus (4.16) gives

$$
\sqrt{\frac{b^{2}}{1+2 \kappa b^{2}}} H_{\alpha \beta}^{*}+b_{i \mid j} B_{\alpha}^{* i} B_{\beta}^{* j}=0
$$

Since $H_{\alpha \beta}^{*}$ is symmetric, it is clear that $b_{i \mid j}$ is symmetric.

Further contracting (4.18) with $v^{\beta}$, we get

$$
\sqrt{\frac{b^{2}}{1+2 \kappa b^{2}}} H_{\alpha}^{*}+b_{i \mid j} B_{\alpha}^{* i} y^{j}=0,
$$

and then with $v^{\alpha}$, we get,

$$
\sqrt{\frac{b^{2}}{1+2 \kappa b^{2}}} H_{0}^{*}+b_{i \mid j} y^{i} y^{j}=0 .
$$

In the view of Lemma 1 , the hypersurface $F^{*(n-1)}(c)$ is a hyperplane of the first kind if and only if $b_{i \mid j} y^{i} y^{j}=0$. Here $b_{i \mid j}$ being the covariant derivative with respect to the Cartan connection of $F^{* n}$ may depend on $y^{i}$.

Since $b_{i}$ is a gradient vector, from (2.11) for induced metric $L^{*}$, we have $E_{i j}^{*}=b_{i j}, F_{i j}^{*}=0$. Thus $(2.12)$ reduces to

$$
\begin{aligned}
D_{j k}^{* i}= & B^{* i} b_{j k}+B_{j}^{* i} b_{0 k}+B_{k}^{* i} b_{0 j}-b_{0 m} g^{i m} B_{j k}^{*}-C_{j m}^{* i} A_{k}^{* m}-C_{k m}^{* i} A_{j}^{* m} \\
& +C_{j k m}^{*} A_{s}^{* n} g^{i s}+\lambda^{s}\left(C_{j m}^{* i} C_{s k}^{* m}+C_{k m}^{* i} C_{s j}^{* n}-C_{j k}^{* m} C_{m s}^{* i}\right)
\end{aligned}
$$

In view of (4.3) and (4.4), the relations in (2.13) become to

$$
\begin{aligned}
B_{i}^{*} & =\left\{2 \kappa+(\epsilon+1)^{2}\right\} b_{i}+\frac{(\epsilon+1)}{\alpha} y^{i}, & & B^{* i}=\frac{2 \kappa}{1+2 \kappa b^{2}} b^{i}+\frac{(\epsilon+1)}{\alpha\left(1+2 \kappa b^{2}\right)} y^{i}, \\
B_{i j}^{*} & =\frac{1}{\alpha} \frac{(\epsilon+1)}{\alpha} a_{i j}-\frac{1}{\alpha^{3}}(\epsilon+1) y_{i} y_{j} & & \\
+ & \frac{6 \kappa(\epsilon+1)}{\alpha} b_{i} b_{j}, & B_{j}^{* i} & =g^{k i} B_{k j}, \\
A_{k}^{* n} & =B_{k}^{* n} b_{00}+B^{* n} b_{k 0}, & \lambda^{* n} & =B^{* n} b_{00} .
\end{aligned}
$$


By virtue of (4.1) we have $B_{i 0}^{*}, B_{0}^{* i}=0$ which leads $A_{0}^{* m}=B^{* m} b_{00}$. Therefore we have

$$
\begin{array}{r}
D_{j 0}^{* i}=B_{j 0}^{* i}+B_{j}^{* i} b_{00}-B^{* m} C_{j m}^{* i} b_{00}, \\
D_{00}^{* i}=B_{00}^{* i}=\left[\frac{2 \kappa}{1+2 \kappa b^{2}} b^{i}+\frac{(\epsilon+1)}{\alpha\left(1+2 \kappa b^{2}\right)} y^{i}\right] b_{00}
\end{array}
$$

Using the relation (4.1), we get

$$
\begin{aligned}
b_{i} D_{j 0}^{* i} & =\frac{2 \kappa b^{2}}{1+2 \kappa b^{2}} b_{j 0}+\frac{(\epsilon+1)\left(1+6 \kappa b^{2}\right)}{2 \alpha\left(1+2 \kappa b^{2}\right)} b_{j} b_{00}-\frac{2 \kappa}{1+2 \kappa b^{2}} b^{m} b_{i} C_{j m}^{* i} b_{00}, \\
b_{i} D_{00}^{* i} & =\frac{2 \kappa b^{2}}{1+2 \kappa b^{2}} b_{00}
\end{aligned}
$$

From (4.12), it follows that $b^{m} b_{i} C_{j m}^{* i} B_{\alpha}^{* i}=b^{2} M_{\alpha}^{*}=0$.

Therefore the relation $b_{i \mid j}=b_{i j}-D_{i j}^{r} b_{r}$, and equations (4.25) and (4.26) give

$$
b_{i \mid j} y^{i} y^{j}=b_{00}-D_{00}^{* r} b_{r}=\frac{1}{1+2 \kappa b^{2}} b_{00}
$$

Consequently (4.19) and (4.20) can be written as

$$
\begin{aligned}
\sqrt{\frac{b^{2}}{1+2 \kappa b^{2}}} H_{\alpha}^{*}+b_{i \mid 0} B_{\alpha \alpha}^{* i} & =0, \\
\sqrt{\frac{b^{2}}{1+2 \kappa b^{2}}} H_{0}^{*}+\frac{1}{1+2 \kappa b^{2}} b_{00} & =0
\end{aligned}
$$

Thus the condition $H_{00}^{*}$ for induced metric is equivalent to $b_{00}$, where $b_{i j}$ does not depend on $y^{i}$. Since $y^{i}$ is to satisfy (4.1), the condition is written as $b_{i j} y^{i} y^{j}=$ $\left(b_{i} y^{i}\right)\left(c_{j} y^{j}\right)$ for some $c_{j}(x)$, so that we have

$$
2 b_{i j}=b_{i} c_{j}+b_{j} c_{i}
$$

Using(4.1), it follows that

$$
b_{00}=0, \quad b_{i j} B_{\alpha}^{* i} B_{\beta}^{* j}=0, \quad b_{i j} B_{\alpha}^{* i} y^{j}=0 .
$$

Again (4.22) and (4.29) gives

$$
b_{i 0} b^{i}=\frac{c_{0} b^{2}}{2}, \quad \lambda^{* n}=0, \quad A_{j}^{* i} B_{\beta}^{* j}=0
$$




$$
B_{i j}^{*} B_{\alpha}^{* i} B_{\beta}^{* j}=\frac{(\epsilon+1)}{2 \alpha} h_{\alpha \beta} .
$$

Using (3.11), (4.4), (4.7), (4.13) and (4.21) we get

$$
b_{r} D_{i j}^{* r} B_{\alpha}^{* i} B_{\beta}^{* j}=-\frac{(\epsilon+1) c_{0} b^{2}}{2 \alpha\left(1+2 \kappa b^{2}\right)^{2}} h_{\alpha \beta}^{*} .
$$

Therefore the relation (4.18) reduces to

$$
\sqrt{\frac{b^{2}}{1+2 \kappa b^{2}}} H_{\alpha \beta}^{*}+\frac{(\epsilon+1) c_{0} b^{2}}{2 \alpha\left(1+2 \kappa b^{2}\right)^{2}} h_{\alpha \beta}^{*}=0 .
$$

Theorem 4.3. The necessary and sufficient condition for $F^{*(n-1)}(c)$ to be hyperplane of 1st kind is (4.29) and in this case the second fundamental tensor of $F^{*(n-1)}(c)$ is proportional to its angular metric tensor.

In view of lemma $(3.3), F^{*(n-1)}(c)$ is a hyperplane of second kind if and only if $H_{\alpha \beta}^{*}=0$. Thus from (4.33), we get $c_{0}=c_{i}(x) y^{i}=0$. Therefore there exist a function $e(x)$ such that $c_{i}(x)=e(x) b_{i}(x)$. Thus (4.29) gives

$$
b_{i j}=e b_{i} b_{j} .
$$

Theorem 4.4. The necessary and sufficient condition for $F^{*(n-1)}(c)$ to be a hyperplane of 2nd kind is (4.34) .

Finally from (4.13) and lemma (3.3) we see that $F^{*(n-1)}(c)$ is not a hyperplane of the 3rd kind.

Theorem 4.5. The hypersurface $F^{*(n-1)}(c)$ of Finsler space with Randers change of General $(\alpha, \beta)$ metric is not a hyperplane of 3rd kind.

\section{References}

[1] P. L. Antonelli, A. Bona and M. A. Slawinski, Seismic rays as Finsler geodesics, Nonlinear Analysis RWA, 5(2003), 711-722.

[2] P. L. Antonelli, R. S. Ingarden and M. Matsumoto, The theory of Sprays and Finsler spaces with Applications in Physics and Biology, Kluwer Academic, Dordrecht, the Netherlands, 1993.

[3] M. Hashiguchi, S. Hojo and M. Matsumoto, On Landsberg spaces of two dimensions with $(\alpha, \beta)$-metric, Journal of the Korean Mathematical Society, 10(1973), 17-26. 
[4] M. Hashiguchi and Y. Ichijiyo, Randers spaces with rectilinear geodesics, Reports of the Faculty of science of Kagoshima University, 13(1980), 33-40.

[5] M. Matsumoto, Projectively flat Finsler spaces with $(\alpha, \beta)$-metric, Reports on Mathematical Physics, 30(1991), 1, 15-20.

[6] M. Matsumoto, Theory of Finsler spaces with $(\alpha, \beta)$-metric, Rep. Math. Phys., 30(1991).

[7] M. Rafie and B. Razaei, On Einstein Matsumoto metrics, Nonlinear Analysis RWA, 63(2012), 882-886.

[8] C. Shibata, On Finsler spaces with an $(\alpha, \beta)$-metric, Journal of Hokkaido University of Education. Section II A, 35(1984), 1, 1-16.

[9] C. Shibata, On Invariant tensors of $\beta$-changes of Finsler metrics, Journal of Mathematics of Koyo University, 24(1984), 1, 163-188.

[10] H. Shimada and S. V. Sabau, Introduction to Matsumoto metrics, Nonlinear Analysis, 63(2005), 165-168.

[11] U. P. Singh and Bindu Kumari, On a hypersurface of a Matsumoto space, Indian J. pure appl. Math., 32(2001), 4, 521-531.

[12] I. L. Yong Lee, Ha-Yong Park and Yong-Duk Lee, On a hypersuface of a special Finsler space with a metric $\alpha+\frac{\beta^{2}}{\alpha}$, Korian J. Math. Sciences, 8(2001), 1, 93-101. 